\title{
Impacto de la implementación de una lista de verificación en procedimientos endovasculares
}

\section{Impact of the implementation of a checklist in endovascular procedures}

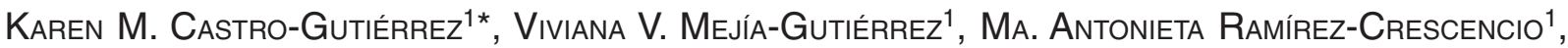 \\ Mirna L. González-Villavelázquez ${ }^{2}$, Alonso Gutiérrez-Romero ${ }^{3}$, Roberto Galán-Galán ${ }^{4}$ \\ y Adolfo Leyva-Rendón 5 \\ ${ }^{1}$ Coordinación de Calidad; ${ }^{2}$ Departamento de Neuroanestesiología; ${ }^{3}$ Departamento de Terapia Endovascular; ${ }^{4}$ Subdirección de Servicios Auxiliares de Diagnóstico y \\ Tratamiento; ${ }^{5}$ Dirección Médica. Instituto Nacional de Neurología y Neurocirugía Manuel Velasco Suárez, Ciudad de México, México
}

\begin{abstract}
Background: The procedures of neurological endovascular therapy are not exempt from complications. At the National Institute of Neurology and Neurosurgery, the number of adverse events associated with these procedures represented $2.41 \%$ of the total reported in 2017. For that reason, a checklist was adapted to the needs of the endovascular therapy praxis, with the aim of adopting it as a safety barrier. Methods: 574 patients undergoing endovascular procedures from April 2018 to July 2019 were included. The data collection was retrospective, and the endovascular procedure checklists registered in the electronic record were used as a data source. The performances of the endovascular procedure checklists and fulfillment of the items were evaluated. Results: Initially, there was an adherence of $62.5 \%$ and a low item's fulfillment; therefore, improvement actions were implemented. At the second audit, the adherence was $79.12 \%$, requiring new actions. The last measurement revealed an adherence of $94.12 \%$ and in general, a greater item's fulfillment. By implementing corrective measures, the adverse events associated to endovascular therapy decreased to 1.88\% of the total reported in 2018. Conclusion: Several actions and three evaluations were made to improve significantly the adherence to items and fulfillment of the checklist, having a positive impact on reducing the adverse events related to endovascular therapy.
\end{abstract}

Key words: Endovascular procedure. Checklist. Adverse event. Patient safety.

\section{Resumen}

Antecedentes: Los procedimientos de terapia endovascular neurológica no se encuentran exentos de complicaciones. En el Instituto Nacional de Neurología y Neurocirugía se observó que el número de eventos adversos asociados a estos procedimientos representó el $2.41 \%$ del total reportado en el 2017 . Por tal motivo, se adaptó una lista de verificación a las necesidades de la práctica de terapia endovascular con el objetivo de implementarla como una barrera de seguridad. Metodología: Se incluyeron 574 pacientes sometidos a procedimientos endovasculares realizados

\section{Correspondence:}

*Karen M. Castro-Gutiérrez

E-mail: karenmelissa.cst@gmail.com
Date of reception: 20-12-2019

Date of acceptance: 30-04-2020

DOI: 10.24875/HMCM.20000257
Available online: 09-07-2020

Hosp Med Clin Manag. 2020;13:48-55

2604-0018 / (c) 2020 Mexican Regional Hospitals of High Specialty and Federal Hospitals. Published by Permanyer. This is an open access article under the CC BY-NC-ND license (http://creativecommons.org/licenses/by-nc-nd/4.0/). 
desde abril del 2018 hasta julio del 2019. La recolección de datos fue retrospectiva y como fuente de datos se utilizaron las listas de verificación para procedimiento endovascular (LVPE) registradas en el expediente clínico electrónico. Se evaluó la realización de las LVPE y el cumplimiento de los apartados. Resultados: Inicialmente hubo un apego del $62.5 \%$ y bajo cumplimiento de los apartados, por lo que se realizaron acciones de mejora. En la segunda revisión el apego fue del $79.12 \%$, requiriendo efectuar nuevas acciones. La última medición reveló un apego del $94.12 \%$ y en general se encontró un mayor cumplimiento de los apartados. A partir de la implementación, los eventos adversos asociados a terapia endovascular disminuyeron al 1.88\% del total reportado en el 2018. Conclusiones: Se hicieron tres evaluaciones y varias acciones que permitieron mejorar de forma significativa el apego y cumplimiento de los apartados de la LVPE, impactando en la disminución del número de eventos adversos relacionados con terapia endovascular.

Palabras clave: Procedimiento endovascular. Lista de verificación. Evento adverso. Seguridad del paciente.

\section{INTRODUCCIÓN}

La terapia endovascular neurológica tiene cada vez mayor auge, ya que es posible realizar procedimientos por medio de navegación intravascular mediante catéteres guiados por fluoroscopia ${ }^{1,2}$. En la actualidad se utiliza en múltiples patologías neurovasculares; sin embargo, no se encuentra exenta de complicaciones (efectos asociados a anticoagulación, reacciones alérgicas, infecciones, etc.) que, en algunos casos, pueden ser catastróficas y llevar a un deterioro neurológico irreversible ${ }^{3-5}$.

Se ha demostrado que una gran proporción de pacientes hospitalizados presenta eventos adversos asociados a procedimientos quirúrgicos, endoscópicos o intervenciones radiológicas ${ }^{6,7}$. La Organización Mundial de la Salud (OMS) ha realizado considerables esfuerzos para reducir el número mundial de defunciones de origen quirúrgico. Una de las iniciativas fue la creación del programa Safe surgery saves lives (La cirugía segura salva vidas) ${ }^{8}$ en el 2008, que consta de un serie de recomendaciones sobre las prácticas de seguridad de la anestesia, infecciones quirúrgicas evitables y la comunicación entre los miembros del equipo quirúrgico.

De este programa surgió la lista de verificación de la seguridad de la cirugía (LVSQ), la cual contribuye a adoptar medidas de seguridad esenciales y a minimizar los riesgos evitables más comunes que ponen en peligro el bienestar y la vida de los pacientes quirúrgicos. Se ha comprobado que el apego a esta lista logra disminuir la tasa de complicaciones del 11 al $7 \%$ y la mortalidad del 1.5 al $0.8 \%$. Debido a la mejora del cumplimiento, la facilidad para su implementación y los logros obtenidos con esta herramienta, su uso se ha propagado rápidamente en el mundo y se considera útil en cualquier institución que proporcione atención médica ${ }^{9,10}$.

De esta manera, el Instituto Nacional de Neurología y Neurocirugía (INNN) comenzó a utilizar la lista propuesta por la OMS en todos los procedimientos de neurocirugía. Sin embargo, se observó que el servicio de terapia endovascular no utilizaba alguna herramienta de verificación al realizar sus procedimientos. Además, el INNN posee un sistema de notificación de eventos adversos y durante la última revisión se encontró un incremento en el número de casos asociados a terapia endovascular, que representaron un $2.41 \%$ del total reportado en el 2017.

Por este motivo, en marzo del 2018 se adaptó una lista de verificación a las necesidades de la práctica del servicio de terapia endovascular, con el objetivo de implementarla como una barrera de seguridad ${ }^{11}$. Este documento consideró los diez objetivos de la OMS para una cirugía segura y las tres fases correspondientes a cada periodo del curso de una intervención: 1) antes de la inducción de la anestesia; 2) antes de la punción arterial o venosa, y 3) antes del egreso de la sala de terapia endovascular ${ }^{12}$.

Para adaptar la lista de verificación de procedimiento endovascular (LVPE) se tomaron en cuenta las características propias del servicio y factores del paciente. Las modificaciones realizadas fueron: la comprobación del uso de anticoagulantes antes del procedimiento, la revisión del funcionamiento óptimo por parte del equipo biomédico, la existencia de material endovascular necesario, la adecuada protección radiológica durante el procedimiento y el requerimiento de condiciones especiales al terminar el 


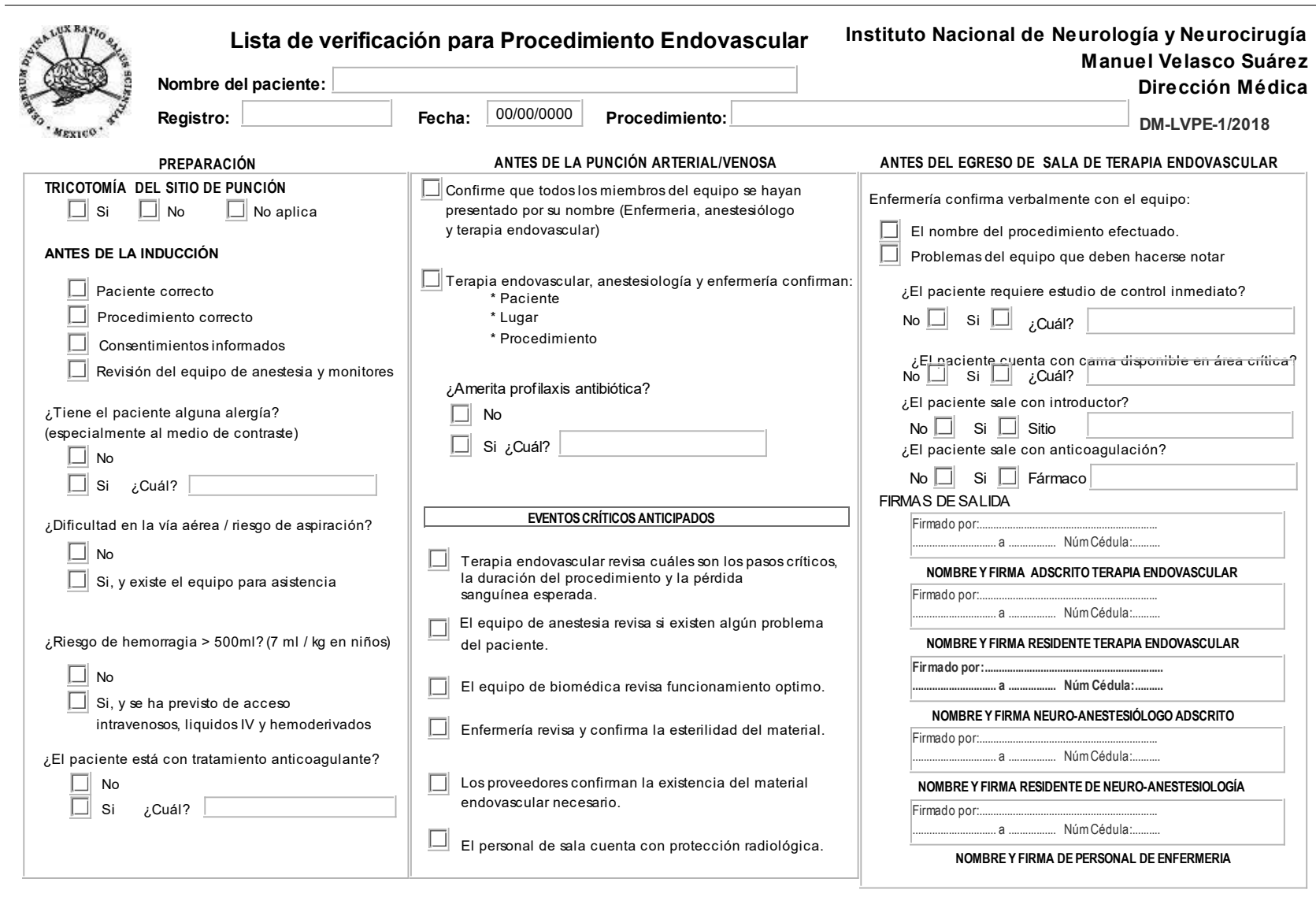

Figura 1. Lista de verificación para procedimiento endovascular del Instituto Nacional de Neurología y Neurocirugía, versión 1,2018 (adaptada de Organización Mundial de la Salud, 2019²0).

procedimiento (estudios de control inmediato, estancia en área crítica, permanencia del introductor o tratamiento anticoagulante) $)^{13}$.

La LVPE (Fig. 1) fue aprobada por el Comité del Expediente Clínico (CEC), por lo que se integró al expediente clínico electrónico a partir de abril del 2018. Se dio capacitación sobre el uso y llenado de la lista al personal de enfermería, neuroanestesiología y terapia endovascular. Además, se colocó un equipo informático en la sala de terapia endovascular para facilitar el llenado oportuno del documento.

Cabe mencionar que en cada procedimiento al menos se encuentran involucrados un médico adscrito de terapia endovascular, un residente de terapia endovascular, un médico adscrito de neuroanestesiología, un residente de neuroanestesiología y un/a enfermero/a. Todos los integrantes del equipo se encargan del Ilenado del formato, ya que los apartados requieren de la participación de las áreas previamente mencionadas.
Antes de realizar el procedimiento se deben llenar los apartados de «preparación», «antes de la punción arterial/venosa» y "eventos críticos anticipados», con el objetivo de registrar alguna situación que deba ser manejada con precaución o contraindique el procedimiento. Al terminar el procedimiento se debe rellenar el apartado «antes del egreso de la sala de terapia endovascular», para hacer énfasis en alguna indicación específica que requiera el paciente. Finalmente, cada una de las personas que participaron en el procedimiento debe de firmar el formato.

\section{Consideraciones éticas}

La auditoría no infringió los derechos, privacidad ni confidencialidad del paciente. No implicó compensaciones entre el costo, la calidad y la seguridad para el paciente. No supuso ningún riesgo o carga para el paciente más allá de la atención habitual. No se obtuvo información del paciente fuera de la rutina. No implicó el uso de intervenciones clínicas o sistemas no probados. 
Tabla 1. Criterios medidos en la lista de verificación de procedimiento endovascular (LVPE)

Evidencia de calidad en la atención o servicio (criterio)

\section{Evidencia de calidad en la atención o servicio (criterio)}

Apego (realización de la LVPE)

Apartados Preparación

Antes de la punción arterial/venosa

Eventos críticos anticipados

Antes del egreso de la sala de terapia endovascular

Firmas de salida

Firmas Firma de salida del médico adscrito de terapia endovascular

Firma de salida del médico residente de terapia endovascular

Firma de salida del médico adscrito de neuroanestesiología

Firma de salida del médico residente de neuroanestesiología

Firma de salida del personal de enfermería
Estándar

(\% de cumplimiento esperado)

\begin{tabular}{ll}
$100 \%$ & Ninguna \\
$100 \%$ & Ninguna \\
$100 \%$ & Ninguna \\
$100 \%$ & Ninguna \\
$100 \%$ & Ninguna \\
$100 \%$ & Ninguna \\
$100 \%$ & Ninguna \\
$100 \%$ & Ninguna \\
$100 \%$ & Ninguna \\
$100 \%$ & Ninguna \\
$100 \%$ & Ninguna \\
\hline
\end{tabular}

\section{Objetivos}

- Adaptar e implementar una lista de verificación de acuerdo con las necesidades y procedimientos de terapia endovascular.

- Evaluar el cumplimiento de la lista en todos los pacientes sometidos a algún procedimiento endovascular.

- Analizar el impacto del uso de la lista en los eventos adversos reportados.

\section{MÉTODOS}

Se incluyeron todos los pacientes sometidos a procedimientos endovasculares realizados en el INNN desde abril del 2018 hasta julio del 2019.

Se realizó una revisión retrospectiva. Al finalizar cada mes se solicitó a los médicos de terapia endovascular la lista de procedimientos que realizaron y el personal de la Coordinación de Calidad se encargó de buscar y evaluar las LVPE registradas en el expediente clínico electrónico en ese periodo.

El primer criterio medido fue la existencia o ausencia del formato en el expediente, es decir, la realización de la lista de verificación en cada procedimiento. Posteriormente, en las LVPE realizadas se evaluó cada apartado, como se muestra en la tabla 1. En todos los criterios se esperó que hubiera un 100\% de cumplimiento, sin excepciones. Para fines de esta auditoría, el análisis se llevó a cabo en tres periodos y se presentaron los resultados en las reuniones del CEC para proponer acciones de mejora que permitieran aumentar el cumplimiento de las listas.

\section{RESULTADOS}

\section{Primer análisis y acciones de mejora}

El primer periodo se evaluó del 10 de abril al 31 agosto del 2018. Se llevaron a cabo 80 procedimientos endovasculares, en los cuales se realizó la LVPE en un $62.5 \%$.

En las 50 listas de verificación realizadas se encontró mayor cumplimiento en los apartados de «preparación» y «antes del egreso de la sala de terapia endovascular», con un 100\% ( $n=50)$, seguido por «antes de la punción arterial/venosa» con un 92\% ( $n=46)$.

Los apartados con menor cumplimiento fueron «eventos críticos anticipados» con un $86 \%(n=43)$ y las firmas de salida con un $32 \%(n=16)$.

También se encontraron diferencias en los perfiles de usuarios que firman, ya que los médicos adscritos de terapia endovascular tuvieron un cumplimiento del $76 \%$ ( $n=38$ ), los residentes de terapia endovascular del $88 \%$ ( $n=44)$, los médicos adscritos de neuroanestesiología del $54 \%(n=27)$, los residentes de neuroanestesiología del 60\% ( $n=30)$ y enfermería del $48 \%$ $(n=24)$.

Se presentaron los resultados en el CEC y se realizó una segunda capacitación que incluyó al personal de 
terapia endovascular, neuroanestesiología y enfermería, con el objetivo de reforzar la importancia y el adecuado llenado de la lista.

\section{Segundo análisis y acciones de mejora}

El siguiente análisis se llevó a cabo del 1 septiembre del 2018 al 28 de febrero del 2019. En este periodo se realizaron 273 procedimientos endovasculares, en los cuales la aplicación del listado en el expediente clínico electrónico se registró en 216 casos (79.12\%).

En este periodo los apartados con mayor cumplimiento fueron «antes de la punción arterial/venosa» con un 94.91\% ( $n=205)$, así como «preparación» y «eventos críticos anticipados», con un $91.67 \%(n=198)$.

Los apartados con menos cumplimiento fueron «antes del egreso de la sala de terapia endovascular» con un $87.96 \%$ $(n=190)$ y las firmas de salida con un $51.85 \%(n=112)$.

En cuanto a las firmas, los médicos adscritos de terapia endovascular cumplieron en un $91.2 \%(n=197)$, un $90.28 \%(n=195)$ de los residentes de terapia endovascular, un $57.87 \%(n=125)$ de los médicos adscritos de neuroanestesiología, un $74.15 \%(n=158)$ de los residentes de neuroanestesiología y un $74.54 \%$ del personal de enfermería.

Como acción de mejora se capacitó a la nueva generación de médicos residentes en el llenado de formatos. También se presentaron los resultados en la sesión de neuroanestesiología para mejorar cumplimiento de las firmas y la Coordinación de Calidad envió un informe de los resultados a los servicios donde se identificó mayor incumplimiento.

\section{Tercer análisis}

El último análisis se realizó del 1 de marzo al 31 julio del 2019. Se llevaron a cabo 221 procedimientos, en los cuales se aplicó la LVPE en 208 casos (94.12\%).

El mayor cumplimiento se encontró en los apartados "antes de la punción arterial/venosa» con un 95.19\% ( $n=198$ ), «eventos críticos anticipados» con un $94.71 \%$ ( $n=197)$ y «preparación» con un $90.38 \%$.

Nuevamente los apartados con menor cumplimiento fueron «antes del egreso de la sala de terapia endovascular» con un $72.60 \%(n=151)$ y las firmas de salida con un $59.13 \%(n=123)$.

De acuerdo con las firmas registradas, los médicos adscritos de terapia endovascular tuvieron un cumplimiento del $95.19 \%(n=198)$, los residentes de terapia endovascular del $92.31 \%(n=192)$, los médicos adscritos de neuroanestesiología del $86.54 \%(n=180)$, los residentes de neuroanestesiología del $88.46 \%(n=184)$ y enfermería del $71.15 \%$.

Al concluir los periodos de evaluación, la Coordinación de Calidad analizó el registro de eventos adversos. Se observó que el uso de la LVPE tuvo impacto al disminuir el número de casos asociados a procedimientos de terapia endovascular. En los reportes del 2017 el 2.41\% estaban relacionados con procedimientos de terapia endovascular. Después de implementar la lista, se encontró un $1.88 \%$ en el 2018.

\section{DISCUSIÓN}

La prevención de los errores médicos evitables ha contribuido en llevar a cabo medidas de seguridad para el paciente con gran énfasis en las listas de verificación ${ }^{14,15}$. Los procedimientos endovasculares conllevan un alto riesgo y algunos de los eventos adversos más frecuentes son complicaciones tromboembólicas, lesiones asociadas con el introductor, ruptura de vasos intraoperatoria y fracaso en el tratamiento de la lesión, entre otras. Por este motivo se han desarrollado estrategias y protocolos para reducir su ocurrencia ${ }^{16}$.

Durante 16 meses se llevaron a cabo 574 procedimientos. Inicialmente el apego y cumplimiento fue bajo (62.5\%), pero conforme la LVPE tuvo aceptación, aumentó al 94.12\% en la última medición (Fig. 2). Al igual que en otros estudios, nos enfrentamos con algunas dificultades para lograr su implementación, ya que existen múltiples factores que pueden impedir el uso adecuado de las listas. Algunos se encuentran relacionados con la organización (recursos, políticas, estándares y metas de seguridad), con los sistemas (protocolos y procedimientos), con el equipo (comunicación, liderazgo y aceptación de la lista) o con la propia checklist (diseño, contenido y aplicabilidad). Por lo tanto, la implementación de una lista de verificación de seguridad es una intervención compleja que requiere la interacción y cooperación de un equipo multidisciplinario ${ }^{17}$. 


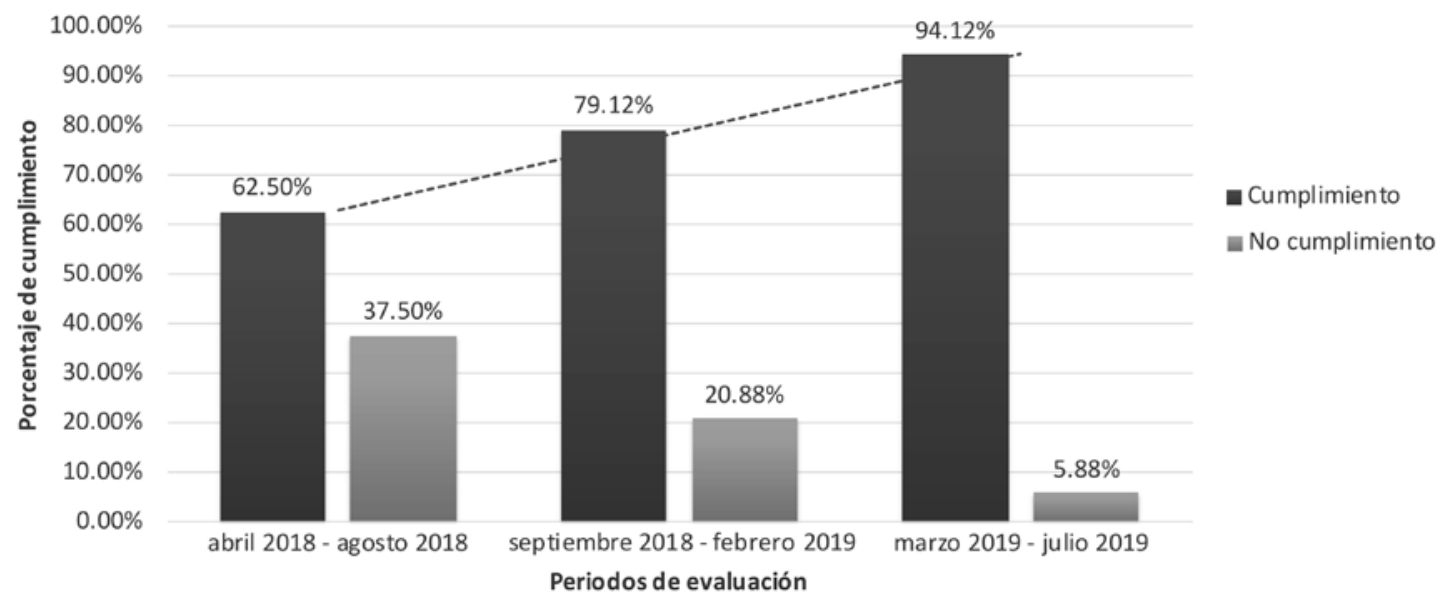

Figura 2. Porcentaje general de cumplimiento de la lista de verificación para procedimientos endovasculares.

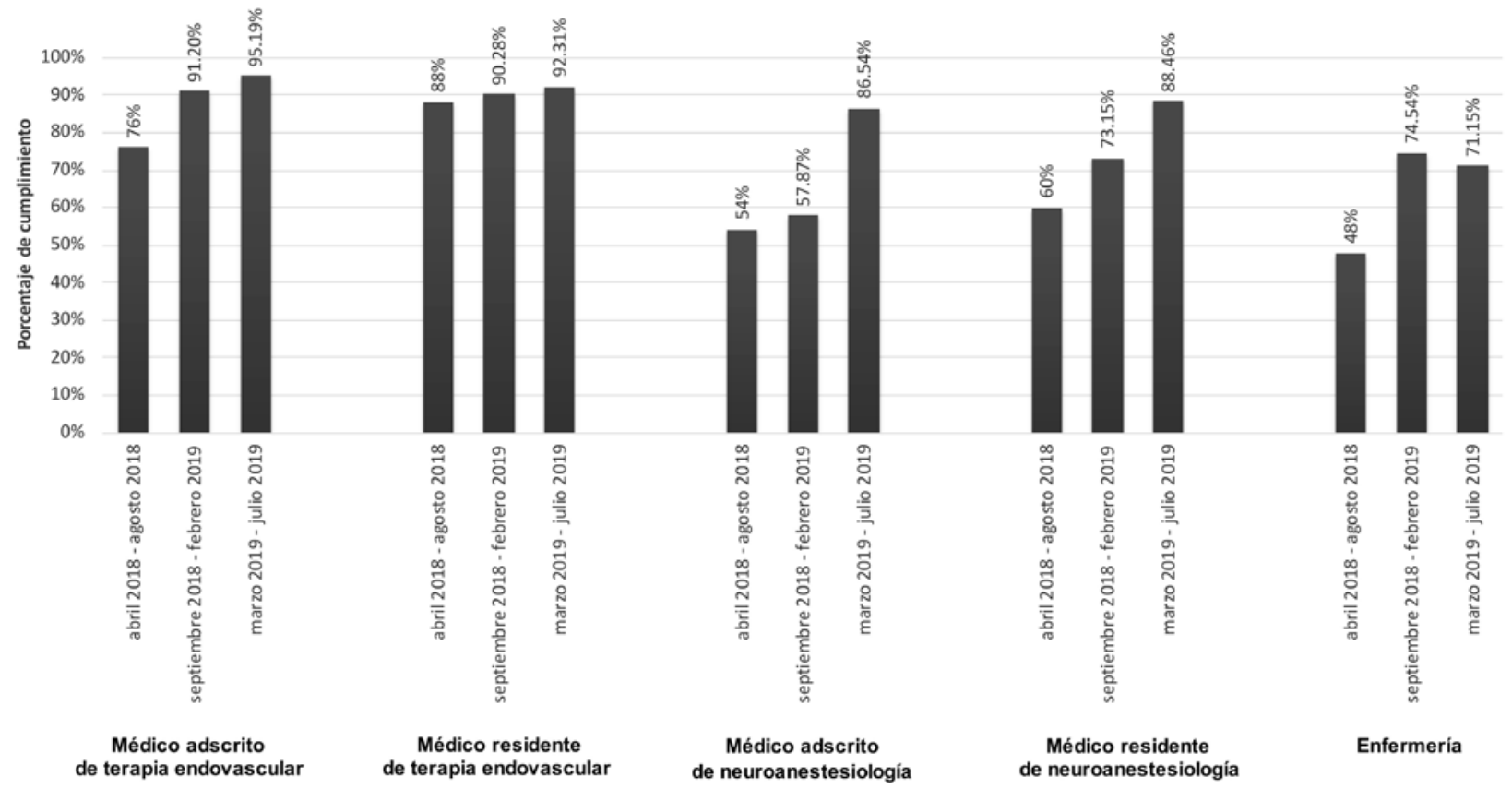

Figura 3. Porcentaje de cumplimiento en las firmas de salida.

Las firmas del personal representaron el principal problema en el llenado de los apartados, principalmente por parte de neuroanestesiología y enfermería (Fig. 3). Esto se podría explicar por la preocupación que genera una posible implicación legal, lo cual también ha sido propuesto por otros autores, mencionando que es un obstáculo para el uso de las listas de verificación ${ }^{18}$. También se ha observado la influencia de la curva de aprendizaje, el cambio de personal en el equipo de trabajo durante procedimientos endovasculares y la resistencia al uso del expediente clínico electrónico. Llama la atención que, aunque el personal de enfermería es quien comúnmente implementa y aplica con mayor compromiso las acciones esenciales para la seguridad del paciente, en nuestra auditoría ha presentado mayor resistencia para el uso del formato y el registro de su firma electrónica.

Por otra parte, el llenado del apartado «antes del egreso de la sala de terapia endovascular» ha tenido una disminución importante, pasando del 100 al 72.60\% (Fig. 4). De acuerdo con los resultados se deberá hacer especial énfasis en este campo, ya que proporciona información valiosa sobre las acciones posteriores y la vigilancia que requiere el paciente, como estudios de control, el uso de anticoagulantes o la permanencia de un introductor. 


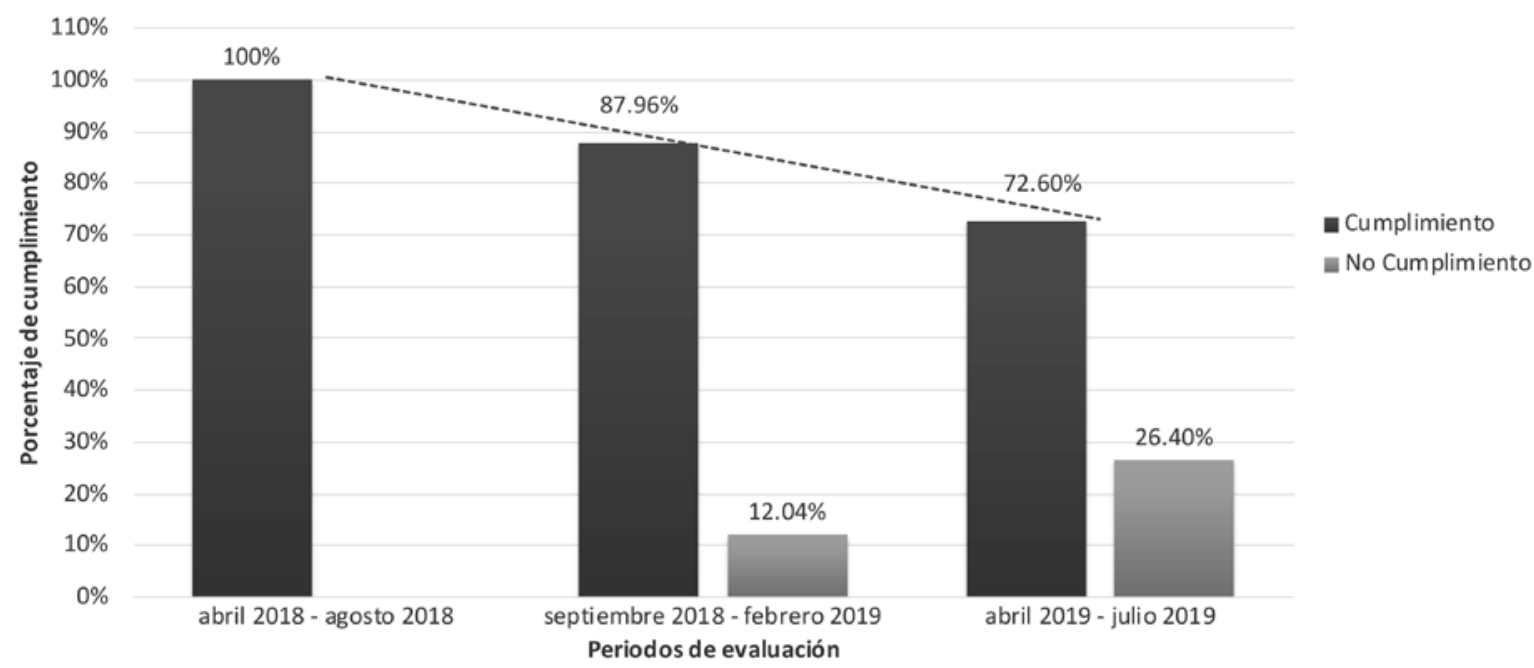

Figura 4. Porcentaje de cumplimiento del apartado «Antes del egreso de la sala de terapia endovascular» de la lista de verificación.

Los eventos adversos se redujeron al $1.88 \%$ en el 2018. Otra publicación también indicó que el uso de una lista de verificación logró reducir la incidencia de sus eventos adversos de un 7.26 a un $3.29 \%{ }^{19}$. En este caso, la severidad de los eventos adversos se relacionó con factores distintos al uso de la LVPE.

Cabe mencionar que no existen auditorías similares en la literatura. Sin embargo, algunos artículos hablan del uso de checklists en radiología intervencionista, los cuales también coinciden en que son una herramienta importante para la comunicación del equipo y asegura que no se olviden los pasos clave en la preparación del paciente, la atención durante el procedimiento y la atención postoperatoria ${ }^{20}$.

Finalmente, la adaptación de la lista de verificación en el servicio de terapia endovascular del INNN contribuyó a que se adoptara con más compromiso y se obtuvieran resultados favorables. A pesar del progreso obtenido, es necesario seguir realizando acciones de mejora para lograr un $100 \%$ en el cumplimiento.

\section{CONCLUSIONES}

Un listado de verificación es una herramienta diseñada para reducir los errores, y el reto es involucrar activamente a todo el personal que interviene en la atención de la salud. Es necesaria la participación de los directivos, jefes de servicio, médicos y personal de enfermería para lograr el objetivo principal, que es la seguridad del paciente.
En el INNN la implementación del listado de verificación en el servicio de terapia endovascular ha tenido aceptación. A partir de las tres evaluaciones y los resultados obtenidos se llevaron a cabo varias acciones que han permitido mejorar de forma significativa el apego y el cumplimiento de los apartados. Además, se ha observado una disminución en los eventos adversos en el servicio de terapia endovascular.

Se seguirán implementando estrategias para que el uso del listado se convierta en una actividad sistemática, con el objetivo de llegar al $100 \%$ de cumplimiento en todos los apartados.

\section{FINANCIAMIENTO}

La presente investigación no ha recibido ninguna beca específica de agencias de los sectores público, comercial o sin ánimo de lucro.

\section{CONFLICTO DE INTERESES}

Los autores declaran no tener conflicto de intereses.

\section{RESPONSABILIDADES ÉTICAS}

Protección de personas y animales. Los autores declaran que para esta investigación no se han realizado experimentos en seres humanos ni en animales. 
Confidencialidad de los datos. Los autores declaran que han seguido los protocolos de su centro de trabajo sobre la publicación de datos de pacientes.

\section{Derecho a la privacidad y consentimiento informado.}

Los autores declaran que en este artículo no aparecen datos de pacientes.

\section{BIBLIOGRAFÍA}

1. Lakhan SE, Kapplan A, Laird C, Leiter Y. The interventionalism of medicine: interventional radiology, cardiology, and neuroradiology. Int Arch Med. 2009;2:27.

2. Jeon $\mathrm{YI}, \mathrm{Kwon} \mathrm{DH}$. Current status and future prospect of endovascular neurosurgery. J Korean Neurosurg Soc. 2008;43:69-78.

3. Sharma MU, Ganjoo P, Singh D, Tandon MS, Agarwal J, Sharma DP, et al. Perioperative complications in endovascular neurosurgery: Anesthesiologist's perspective. Asian J Neurosurg. 2017;12:6-12.

4. Osorio MA, González ML, Obregón A. Manejo anestésico en terapia endovascular neurológica. Rev Mex Anestesiol. 2012;35:143-7.

5. Lee CZ. Anesthesia for interventional neuroradiology. Rev Colomb Anestesiol. 2015;43:151-5.

6. Vries EN, Ramrattan MA, Smorenburg SM, Gouma DJ, Boermeester MA. The incidence and nature of in-hospital adverse events: a systematic review. Qual Saf Health Care. 2008;17:216-23.

7. Vázquez A, Luque JM, Nozal M, Barroso C, Román M, Vilaplana A. Efectividad de una intervención para mejorar la cumplimentación del listado de verificación de seguridad quirúrgica en un hospital de tercer nivel. Rev Calid Asist. 2016;31:24-8.

8. Lista de verificación de seguridad de la cirugía (1. a edición) [Internet]. Organización Mundial de la Salud; 17 de septiembre de 2019. Disponible en: https://apps.who.int/iris/bitstream/handle/10665/70083/WHO_IER_
PSP 2008.05 Checklist spa.pdf;jsessionid $=$ 52944EF5ECCOC1D328CB49 7E07BD9FF8? sequence $=2$

9. Haynes AB, Weiser TG, Berry WR, Lipsitz SR, Breizat AH, Patchen E, et al. A surgical safety checklist to reduce morbidity and mortality in a global population. N Engl J Med. 2009;360:491-9.

10. López E, Arroyo JY, Zamora AA, Montalvo A. La implementación de la lista de verificación para una cirugía segura y su impacto en la morbimortalidad. Cir Gen. 2016;38:12-8.

11. Tsetis D, Uberoi R, Fanelli F, Robertson I, Krokidis M, van Delden O, et al. The provision of interventional radiology services in Europe: CIRSE Recommendations. Cardiovasc Intervent Radiol. 2016;39:500-6.

12. Cote L. Lista de verificación, protocolo universal, tiempo fuera. ¿Qué sigue? Cir Gen. 2011;33:99-102.

13. Rafiei P, Walser EM, Duncan JR, Rana H, Ross JR, Kerlan RK, et al. Society of Interventional Radiology IR Pre-Procedure Patient Safety Checklist by the Safety and Health Committee. J Vasc Interv Radiol. 2016;27:695-9.

14. Zuckerman SL, Fargen KM, Mocco J. Neurosurgical checklists. Neurosurg Clin N Am. 2015;26:219-29.

15. Solor A, Pérez L. El checklist como herramienta para el desarrollo de la seguridad al paciente quirúrgico. SCAR. 2015;14:50-7.

16. Wong JM, Ziewacz JE, Panchmatia JR, Bader AM, Pandey AS, Thompson $B G$, et al. Patterns in neurosurgical adverse events: endovascular neurosurgery. Neurosurg Focus. 2012;33:1-8.

17. Russ SJ, Sevdalis N, Moorthy K, Mayer EK, Rout S, Cais J, et al. A qualitative evaluation of the barriers and facilitators toward implementation of the WHO Surgical Safety Checklist across hospitals in England: lessons from the "Surgical Checklist Implementation Project". Ann Surg. 2015;261:81-91.

18. Bergs J, Lambrechts F, Simons P, Vlayen A, Marneffe W, Hellings J, et al. Barriers and facilitators related to the implementation of surgical safety checklists: a systematic review of the qualitative evidence. BMJ Qual Saf. 2015;0:1-11.

19. Collazos C, Bermúdez L, Quintero A, Quintero LE, Díaz MM. Verificación de la lista de chequeo para seguridad en cirugía desde la perspectiva del paciente. Rev Colomb Anestesiol. 2013;41:109-13.

20. Wong SS, Cleverly S, Tan KT, Roche-Nagle G. Impact and culture change after the implementation of a preprocedural checklist in an interventional radiology department. J Patient Saf. 2015;0:1-4. 